\title{
Self-Organising Map Analysis of Seismicity Associated with Mining at Mount Charlotte Mine
}

\author{
P.A. Mikula Mikula Geotechnics Pty Ltd, Australia \\ S.J. Fraser CSIRO Exploration and Mining, Australia
}

M.F. Lee AMC Consultants Pty Ltd, Australia

\begin{abstract}
Seismicity data from the mining of the ROB5 stope at Mount Charlotte mine is analysed using an approach known as self organising maps (SOM). SOM is an established technique that can reveal relationships between variables in complex and diverse data sets in an objective fashion. However, the mining industry has generally not recognised the potential of the technique. SOM is unsupervised - no prior knowledge is required as to the nature or number of groupings within the data set.

The SOM approach is complementary to the MS-RAP spatial clustering and seismicity analysis package used at Mount Charlotte mine. SOM can form clusters based on spatial position, but its particular strength is its ability to cluster on the basis of any set of user-defined seismic event attributes, not just on location.

This paper describes SOM clustering based on location, magnitude and the time interval between successive events. For the Mount Charlotte analyses, the absolute time of the seismic events was not revealed to the SOM. Yet, unassisted, SOM identified a clear change in seismic behaviour in June 2007 which correlated with the last major seismic event affecting the ROB5 destressing sequence. It also identified post-blast trends that may assist forecasting of increasing or decreasing seismicity. SOM also identified trends suggesting that specific lengths of seismic quiescence preceded higher magnitude events around the ROB5 stope.
\end{abstract}

\section{Introduction}

SOM is a data mining technique that can reveal relationships between and amongst variables in complex and diverse data sets in an objective fashion. An enhanced version of SOM developed by the CSIRO is called SiroSOM. It is used in this study for a novel purpose, namely the trial analysis of a seismicity dataset associated with destressing and mining the highly stressed ROB5 remnant at Kalgoorlie Consolidated Gold Mines (KCGM) Mount Charlotte gold mine at Kalgoorlie.

The motivation was to advance the understanding of mining-induced seismicity for a stope that has previously been well-documented and reported using conventional analysis techniques (Mikula et al., 2005; Kempin et al., 2007).

The main objective of analysing mining-induced seismicity is to understand when, where and why it occurs, especially in relation to the larger events. The practical aim is to prevent the large events occurring, or if this is not possible, establish systems and procedures to provide a safe operating environment for the workforce.

The data available to the geotechnical engineer to manage seismicity includes:

- Technical knowledge, often based on experience or numerical modelling, about relationships between stopes, structures, stresses and seismicity.

- Guidelines on appropriate stope designs and sequences for mining in seismically active ground.

- The seismic event dataset, an understanding of which can be used to improve the above two points via feedback. While gross seismicity patterns are often self-evident, the exact details can be very confusing; but a very good understanding of the details is critically important to providing a safe work place. SOM can assist the identification of subtle connections and reasons behind patterns of observed seismicity. 
The preliminary SOM investigations reported in this paper focussed on two main issues:

- Investigating links between blasts (initiating factors) and seismic events (consequences) in terms of magnitude, spatial coordinates and timing. The objective was to develop a better understanding of when and where large events occur.

- Searching for conditions under which large events occurred; i.e. anything that might characterise or fingerprint them.

As a preliminary study, this work has shown the usefulness and applicability of the SOM approach to a complex data set, and it should encourage further application of the technique within the mining industry. Alongside standard and more conventional investigations, SOM can assist in the formulation of a better seismic knowledge base for mines.

\section{Definitions}

Node: A collection of similar seismic events made by SOM.

Cluster: A collection of similar seismic events made by MS-RAP.

Group: A collection of similar SOM nodes or MS-RAP clusters.

\section{What is SOM?}

SOM is a data analysis tool that allows relationships between variables and samples to be determined in an objective unbiased manner. It was developed in the 1980s by T. Kohonen (Kohonen, 2001), and since then about 250 papers have been published every year using the method.

SOM has been widely used for data analysis in fields including finance, industrial control, speech analysis, astronomy, and petroleum exploration. Apart from the oil industry, which has used SOM in seismic and well-logging applications, and a few workers in the multi-spectral and remote sensing field, the earth sciences, including the mining industry, has generally not yet recognised the potential of the technique to mine its large and often complex data sets.

Exploration and mining companies typically acquire and assess vast amounts of spatially located geological, geochemical, geophysical and geotechnical data. Data is typically gathered at a rate faster than it can be interpreted. There is a need for enhanced computational methods and technologies to improve the efficiency and effectiveness of geoscientists to identify often subtle clues and relationships in large, complex and diverse data sets. SOM is potentially such a method.

The SOM algorithm generates a two-dimensional rectilinear map that represents the underlying structure, or essence of the multivariate data set. SOM outputs are highly visual displays that allow the identification of trends within the data. Using seismic event parameters for illustration, SOM has the following advantages:

- Versatile: It is able to deal with both continuous input values (e.g. the magnitude of each event) and categorical input values (assigned or named, e.g. yes or no regarding whether the event was near a fault).

- Unbiased: Because the SOM analysis is unsupervised, no prior knowledge is required as to the nature or number of groupings within the data set. Normally, one would attempt to analyse seismic events by grouping them by obvious parameters such as geometry, time or energy level. Acting unsupervised, SOM does both; it forms its own groupings and allows further analysis of them.

- Adaptable: If new data variables, or more data, become available, SOM has the ability to change.

- Robust: It can also deal with an incomplete database (e.g. do not know whether an event was near a fault), or with noise (e.g. erroneous parameter values).

- Scaleable: Both linear and non-linear relationships can be represented and revealed amongst data variables. 
Perhaps the most novel aspect of SOM, for the investigation of seismic parameters, is its ability to perform non-spatial clustering. Practitioners are familiar with the concept of seismicity groups occupying a certain spatial area or domain. SOM groups, however, can occupy any user-defined domain in multivariate space; e.g. magnitude, energy, S:P Energy Ratio, or any combinations of available parameters.

These features make SOM attractive when compared with more conventional analysis methods, such as clustering (both hard and fuzzy), factor analysis, neural networks and principal components.

\section{Seismicity data analysis at Mount Charlotte}

A seismicity analysis tool used regularly at Mount Charlotte is MS-RAP. This is a package developed by the ACG with industry funding, and used in many Australian mines (Hudyma et al., 2007). MS-RAP performs statistical analyses, estimates peak particle velocity (PPV) and seismic hazard levels, and presents graphical displays of seismic events based on clusters and groups.

The division of seismicity into groups by MS-RAP is on the basis of spatial position. Characteristics of these groups are charted for further analysis. A major aim of MS-RAP is to make clustering, charting and statistical reporting easy for site practitioners, so that they can spend more time on analysis of trends and evaluating risk. Operationally, MS-RAP is very useful. PPV forecasts are a key output, being required to estimate adequate support capacity for seismic conditions.

However, presently there are no recognised tools to quantify the underlying relationships that dictate the character of seismic zones; i.e. to identify (without supervision) the subtle connections and reasons behind the patterns of observed seismicity. Empirical relationships have been developed at Mount Charlotte over numerous years of observations, investigations and contemplation, and have evolved into a set of sitespecific rules as an aid to mine design in seismic ground (Mikula and Lee, 2002). These rules advocate, for instance, not mining towards a major fault.

Compilation of all the rules and associated knowledge forms the seismic knowledge base (SKB) for the mine. SOM may eventually allow the automated (unsupervised) formulation of rules, which would be added to the mine's SKB.

\section{Previous Mount Charlotte SOM experience}

SiroSOM was trialled on a Mount Charlotte database in 2004 (Fraser, 2004; Fraser et al., 2006). It was used to investigate the mine's existing empirical seismic risk rules in relation to large seismic events, and found that the experience-based rules unfortunately had variable and sometimes poor correlation with large events. Indeed this illustrated the difficulty in arriving at rules based on experience, especially when many conflicting parameters are involved.

The 2004 trial was not progressed beyond the initial findings. At the time, the future of the mine was uncertain and the proposal to mine the ROB5 remnant was being debated.

\section{Mount Charlotte ROB5 background and dataset}

To demonstrate the capabilities of SOM for seismicity analysis, a relatively small, compact and wellunderstood seismicity database was needed. Seismicity associated with the mining of the ROB5 remnant at Mount Charlotte during 2006-2007 represented just such a database. As nearly all the seismicity occurred while only ROB5 was being mined, the database can be regarded as closed.

The current SOM investigation has benefited from excellent prior documentation of the ROB5 remnant mining, including numerical modelling of the destressing concept (Sharrock, 2004), and stope design, seismicity analysis, ground behaviour and ground support design (Mikula, 2006). While a range of conclusions were reported about interactions between stoping, stresses, structures and seismicity for the ROB5 remnant, this was a difficult task. A considerable amount of highly variable and sometimes inaccurate or incomplete data had to be assessed. But with that background and understanding in place, a SOM analysis of the seismicity associated with mining the ROB5 remnant was attractive. 
The data was treated as a complete record of seismicity; it covered all the remnant mining, and the stope was significantly distant from other mining areas at Mount Charlotte. The ROB5 remnant was, by far, the major seismically active area in the mine.

The event dataset was cleaned by removing events that were unclustered by MS-RAP; about $10 \%$ of events. Practitioners who use MS-RAP have found that most events labelled by MS-RAP as unclustered are due to errors in processing, or relate to an event well outside of the sensor array. Their locations and source parameters are therefore suspect. For the ROB5 dataset, such events were typically remote from the ROB5 remnant stoping (an average of $445 \mathrm{~m}$, but up to $2.3 \mathrm{~km}$ away), and they had high location errors (over $50 \mathrm{~m}$ ). Also removed were events generally over $400 \mathrm{~m}$ from the ROB5 remnant area, including events related to mining in the 1302 and 2320 mining areas.

The final dataset comprised 6121 seismic events with magnitude, time, location and several source parameters. Stope blast date/time information was also included in the dataset.

\section{$7 \quad$ Introduction to SOM maps and plots}

SOM colour output displays comprise K-means clusters plots, component plots, and cross-plots, with examples shown in Figures 1 and 2.

The self organised map is a two-dimensional representation of an n-dimensional data set, in which:

- Each node represents a number of similar samples (for the seismicity analysis in this paper, a node represents a collection of seismic events having similar characteristics).

- Adjoining nodes are topologically-ordered on the map surface.
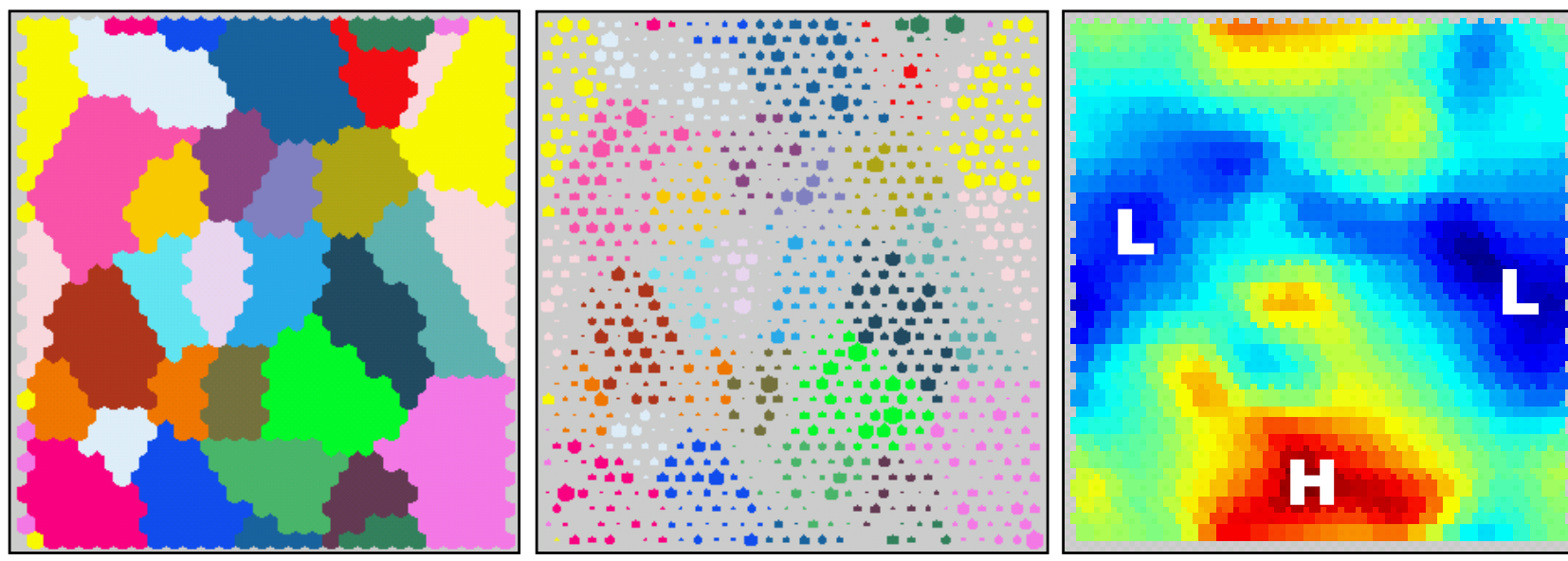

Figure 1 Left: The SOM K-means cluster diagram of the map for the ROB5 event dataset. Each coloured area represents a group of similar nodes. Centre: The arrangement of the populated nodes in the map. The size of each node is proportional to the number of similar seismic events represented. Right: A component plot, in this case of magnitude. Warmer colours (browns) indicate high (H) values; cool colours (blues) indicate low $(\mathrm{L})$ values. The highest magnitudes are associated with the dark-blue and pale-green groups at the bottom centre of the K-means cluster diagram. Map edges wrap top to bottom and side to side

SOM is an averaging method, so for the ROB5 work each node represents the average parameter values of a collection of events. Individual outlier data for specific events is not shown or plotted, but can be ascertained via a quality error parameter.

The K-means clusters representation of the map (Figure 1) shows the assignment of the SOM nodes into larger groups that have similar characteristics. Granularity refers to the scale of how SOM groups the nodes. The finest granularity would be to display events based on an individual node for each seismic event. Granularity is coarsened upwards with a decreasing number of K-means Clusters. MS-RAP practitioners do the same manually when they allocate MS-RAP clusters into larger groups. 
Each coloured node represents a collection of events, not individual events, and several like-coloured nodes represent a larger grouping of events that have similar parameters. Thus, by referring to the group colour and its position in the component plots, various parameters can be assessed. It becomes clear what characteristics fit which groups.

The random seeding process used by SOM means that group positions and colours are unique to each SOM run. It is not valid to compare colours between plots from different SOM runs.

Cross-plots of any two variables can be generated (Figure 2). On these, the dots are the SOM nodes, not individual events and they are sized according to the number of events each node represents. Larger dots imply a more significant group of similar seismic events.

To link into empirical seismicity knowledge about the ROB5 stoping, seismic event spatial location (N, E and Elevation) was included in all of the SOM analyses.

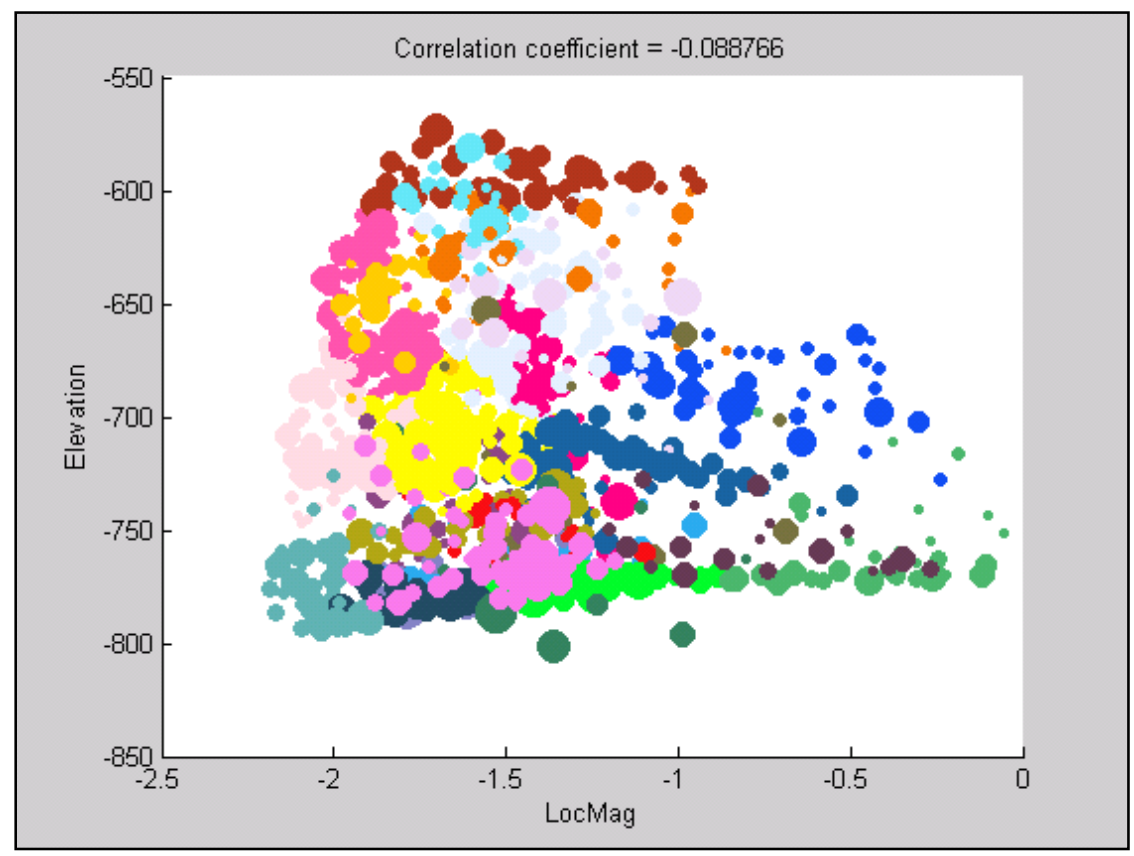

Figure 2 SOM cross-plot of magnitude versus elevation. All the nodes shown in Figure 1 (centre) are plotted by their average magnitude and elevation, retaining their colour and size attributes from Figure 1. Elevation in m; LocMag is approximate Richter magnitude

\section{Comparison of SOM and MS-RAP spatial clustering}

The SOM procedure makes use of similarities and differences in all the parameters chosen to be included in the analysis. Thus if depth, for instance, is an included parameter, the SOM will distinguish deeper from shallower events. If magnitude is included, high and low magnitude events will be separated (i.e. a certain SOM group may contain only large events); and if time is included, the resulting SOM groups will be related in terms of timing.

This is a key contrast with MS-RAP clusters, in which neither event magnitude nor timing is considered during the clustering process. The MS-RAP clusters may include events with widely differing magnitudes and times of occurrence. MS-RAP approaches clustering by using rules to find a denser volume of events, then initiating a cluster, then searching specified nearby space for additional events that are not part of another cluster. Anything left over remains unclustered. Users then inspect the clusters and manually define larger groups by combining clusters, parts of clusters and/or selected individual events, with the option of taking event timing into account.

For each MS-RAP cluster or group, the seismic event magnitudes fit the Gutenberg-Richter diagram, and information can be derived about magnitudes and other characteristics of that group. If SOM is run with just 
the spatial data, the resulting SOM groups are similar to the MS-RAP groups, and the SOM groups could be similarly analysed using Gutenberg-Richter principles.

It is important to note that as soon as time, magnitude or related parameters are added into the SOM analysis, the SOM groups also reflect those parameters and immediately differ from the MS-RAP groups. Indeed SOM can be asked, for example, to define groups containing events that are truly similar in all parameters other than spatial, and then show how these groups are arranged in space. However, the Gutenberg-Richter diagram is then not applicable to such groups.

\subsection{Spatial clustering comparison test}

SOM was directed to perform clustering of the ROB5 event data on the same basis as MS-RAP by choosing only spatial coordinates as the parameters for the SOM analysis. All other parameters, such as time and magnitude, were excluded from this analysis.

A 7200 node self organised map was constructed for this comparison. This map had room for every event (of the 6121 events) to be represented by a unique node. However, the events self-organised onto 4182 nodes, leaving 3018 nodes empty. An analysis of the MS-RAP event cluster-coding from the samples on each of the populated nodes revealed that there were 222 event-samples that were on a node where the MS-RAP clustercoding differed from the predominate MS-RAP cluster-coding for that node. That is, about $3.6 \%$ of the input event samples as clustered by MS-RAP doubled up on SOM nodes with events with a different MS-RAP cluster-code. SOM could not distinguish or separate the double-ups based on their location.

The double-ups suggest that MS-RAP has allocated spatially-similar events into more than one MS-RAP cluster. This is indeed possible. In the practical operating environment of mining, there will always be a degree of manual involvement in seismic data processing. Engineers may revise event locations within MSRAP, or may inadvertently mis-assign certain events or clusters. Also, as MS-RAP clusters grow over time, they extend and may in part intertwine with adjacent clusters; like growing crystals. Periodic review and maintenance of the MS-RAP clusters is required.

This comparison shows that the SOM and MS-RAP approaches differ slightly. Because SOM is an objective data-driven technique, it is possible that the SOM analysis is highlighting the degree to which the MS-RAP manual processing is subjective. On this basis, it is possible that SOM may have a slight advantage.

\section{SOM spatial, magnitude and time clustering}

Care must be taken when including spatial and timing parameters in a SOM analysis because of the bias they can introduce. The SOM analyses in this paper typically include the spatial parameters (N, E and Elevation). Most of the analyses include the non-spatial parameter magnitude and some use another non-spatial parameter, the time difference between events (DeltaT). The time (DateValue) parameter was typically excluded from analyses (but used to display trends of SOM outputs over time), and included only in special circumstances.

Because of the additional SOM input parameters, the groups are not simply spatial. Similar events need not be very close together in space; they can be more wide spread - SOM finds them.

Figure 3 shows details of an example grouping and it also shows and explains the component plots for this analysis. Component values range from high (reds and browns) to low (blues). Group position in the component plots relates directly to component values, as shown for instance, for the example green group (circled). By inspection, it is seen that this group is characterised by:

- Low-moderate northing/moderate-high easting/low elevation (more negative or deeper).

- Moderate DateValue (mid-stoping dates).

- Low to moderate magnitudes. 


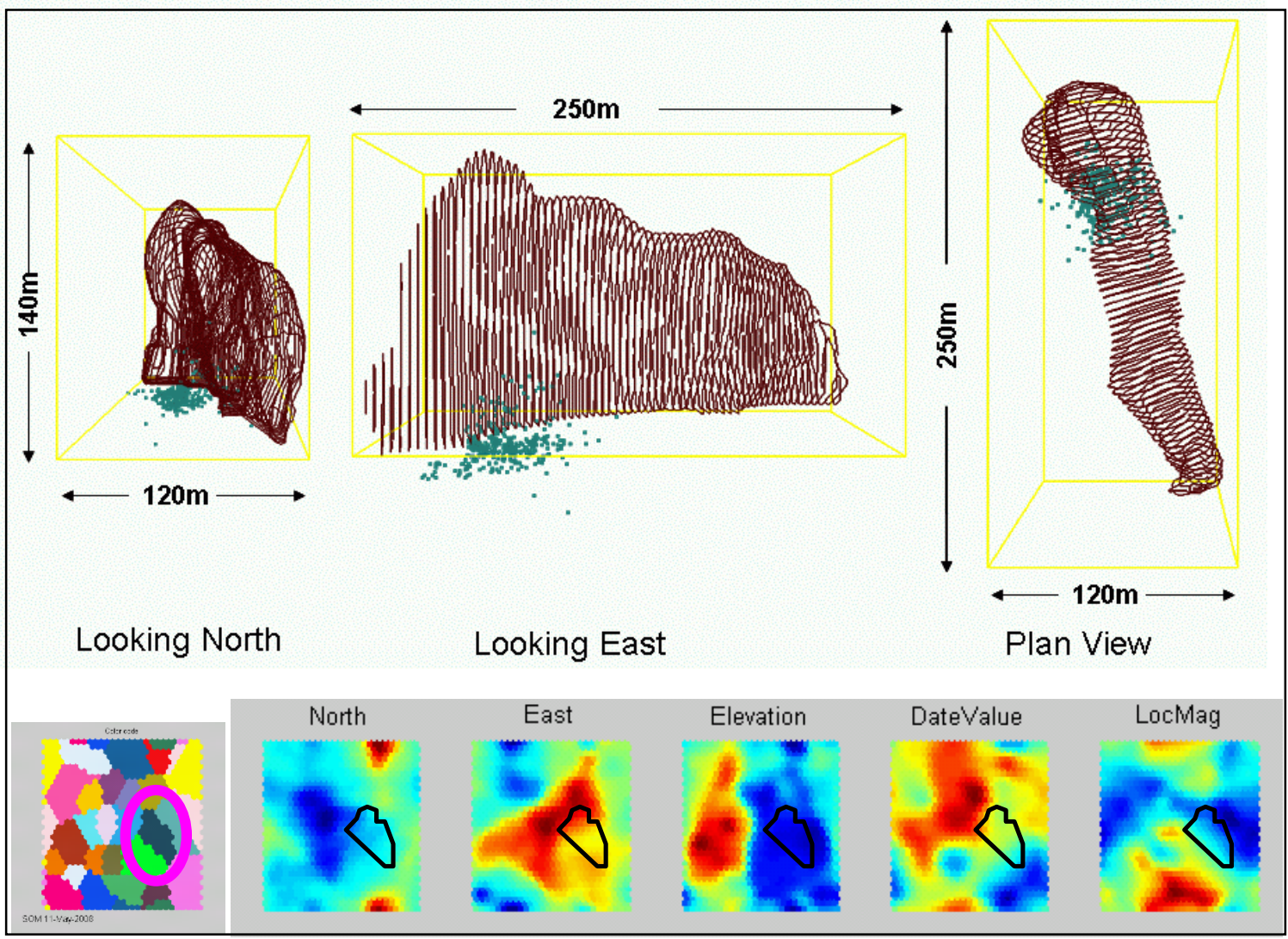

Figure 3 (Above) Example of an individual SOM-derived group of events, showing event locations in space compared to the ROB5 stope wireframe outline. (Below) Also shown is the position of the group highlighted on the K-means cluster plot (left-hand plot), and in the component plots (north, east, elevation, DateValue and LocMag). Refer Figure 1 for Kmeans legend

\section{SOM time-series analysis of ROB5 seismicity}

Time-series data have been used for other SOM applications with good results. The strategy is to exclude the event DateValue (date/time) from the actual analysis, but to use a DateValue axis to plot results. Nodes are plotted according to their real times, but the nodes themselves are based on parameters other than time.

Meaningful trends in ROB5 seismicity were found as shown in Figure 4, and in other cross-plots not shown here. Figure 4 shows that seismic characteristics have changed as mining progressed. Although the date/time of events was not revealed to the SOM analysis, the SOM groups respect time. This demonstrates the power of SOM to recognise the trends in other parameters, in this case magnitude, north, east and elevation, that have occurred over time as the ROB5 remnant was mined.

In Figure 4, the magnitude of the mid-green nodes gradually increases with time. This group becomes more hazardous with time, but in a forecastable fashion. The mid-green nodes were found to associate with seismicity on the west-dipping Shea fault. In contrast, the dark-yellow group (D) starts with high magnitude events (high initial seismic hazard), but its magnitude decreases over time. This group includes some events in the abutment area below the destressing slot in the stope. These observations invite a deeper study of the properties of those two groups. It is important to identify the characteristics of particular groups to appreciate their effect on seismic hazard.

The highest magnitude nodes in Figure 4 occur at about day 39,250. A significant seismic event occurred with a blast on 4 June 2007 (day 39,237), causing some damage (see Figure 5). At the time, it was wondered whether, or not, the event represented the final major movement that effectively completed the destressing of the area. SOM demonstrates that it did. 
Coincidentally at about the same time, relaxation was noted of a very large volume of rock on the west side and above the stope, characterised by numerous small events on west-dipping faults and structures. This activity is represented by the yellow group (Y) in Figure 4.

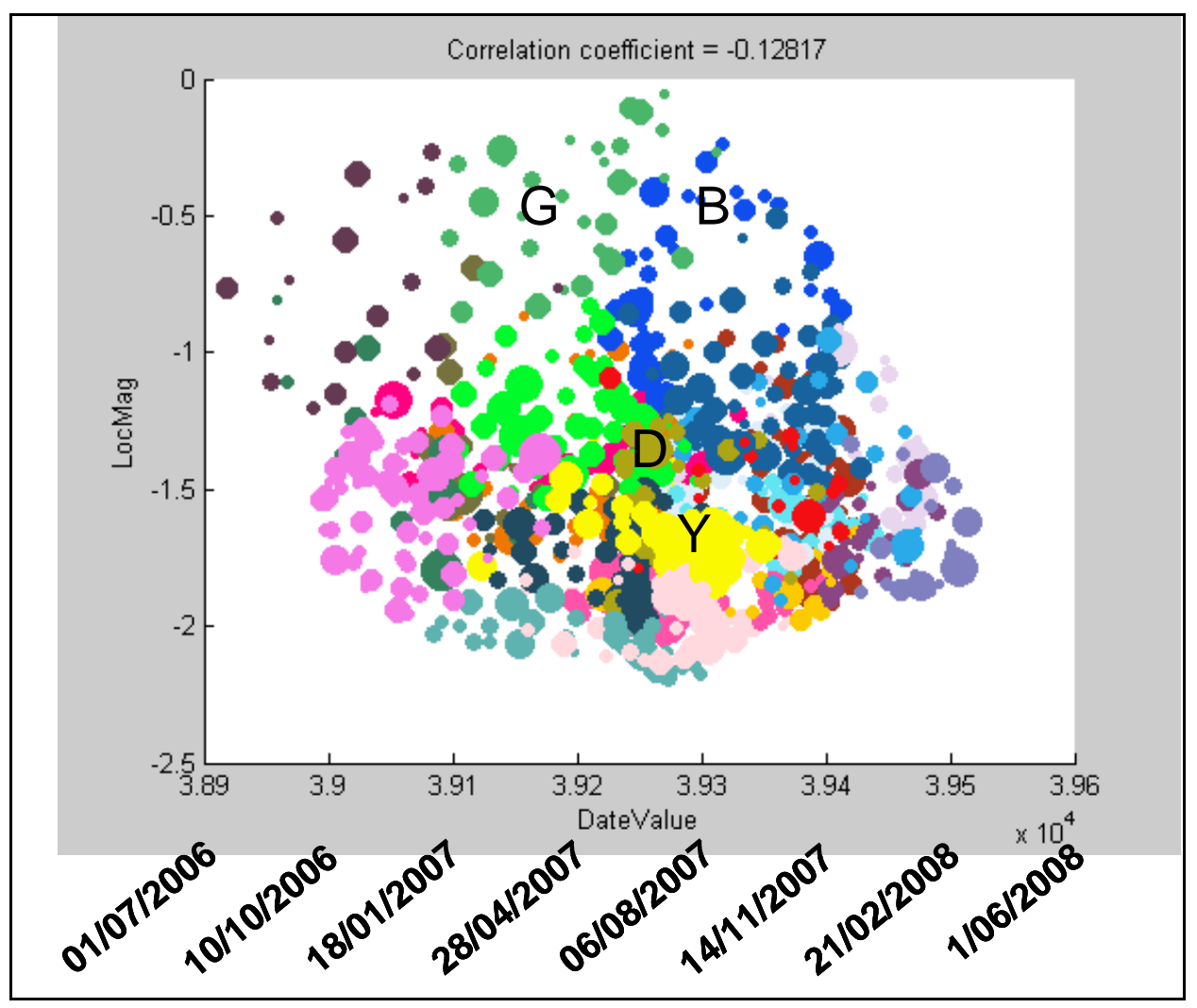

Figure 4 Cross-plot of magnitude versus time. SOM has successfully separated nodes by time, without being given the time data. Node magnitude peak is about day 39,250 (June 2007), then falling sharply with time as the green group $(G)$ vanishes to be replaced by the blue group (B). Refer text for comment on yellow (Y) and dark yellow (D) groups. Magnitude is approximate Richter

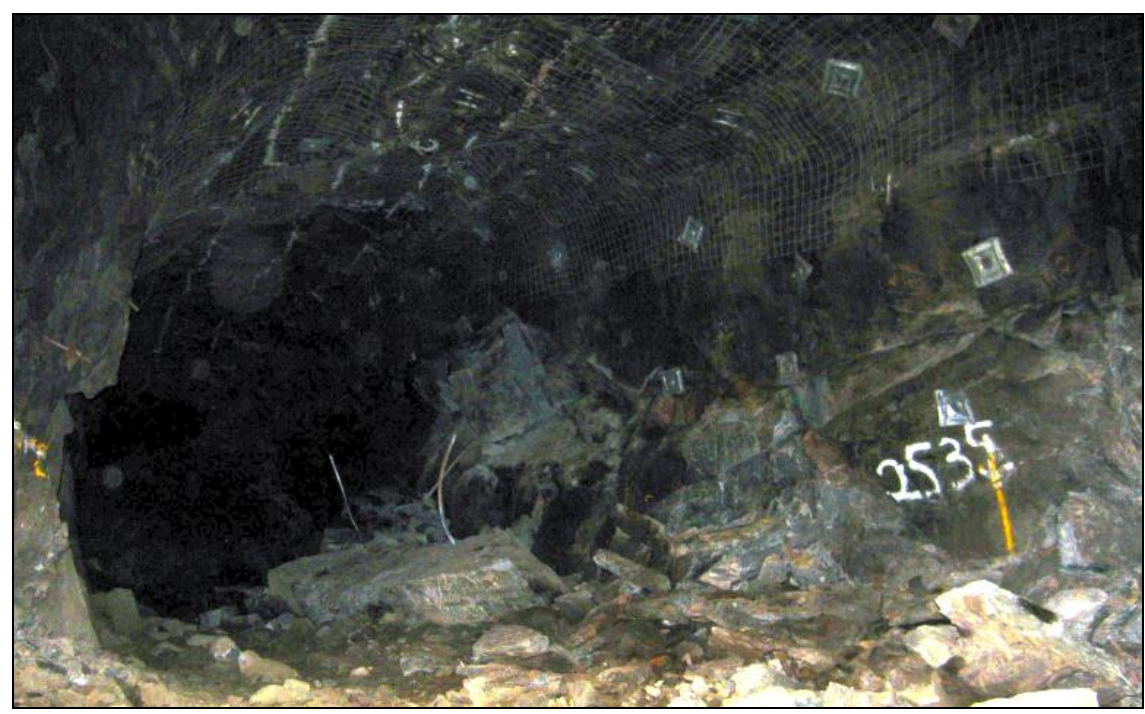

Figure 5 Damage in the ROB5 2535 drive associated with a Richter magnitude 1.7 seismic event following a blast on 4 June 2007 


\section{SOM analysis of events after blasts}

Two ROB5 blasts, selected from the 122 available, were analysed using SOM; see Table 1. SOM was used to study post-blast seismic patterns until the next major event or the next blast. This assesses the same dataset as is typically used to estimate re-entry times using the Omori method, but the SOM analysis is different as magnitude and location parameters were included.

Table 1 Selected ROB5 blasts analysed using SOM

\begin{tabular}{lll}
\hline Blast Date & Location & Comment \\
$\begin{array}{l}\text { 1 November } \\
2006\end{array}$ & $\begin{array}{l}\text { 2515 RAR; } \\
\text { m/h A rings } \\
104-106\end{array}$ & $\begin{array}{l}\text { Early in the mining cycle. This blast removed the intersection of MH A } \\
\text { and the RAR rise. Only about } 2 / 3 \text { of the rise was fired successfully, } \\
\text { leaving a bridge near 24 Level. The area was not destressed. The overall } \\
\text { stress field was not greatly affected up to this stage in the mining. } \\
\text { Much later in the mining cycle. After about } 100 \text { m southward advance of } \\
\text { the destressing slot. Two large seismic events had occurred in the previous } \\
\text { four months, causing destressing in the slotted area and stress build-up in } \\
\text { the area of this blast (the slot southern abutment). }\end{array}$ \\
3 May 2007 & $\begin{array}{l}\text { 2515 S5 ring } \\
538\end{array}$
\end{tabular}

\subsection{Variation of source corner frequency (SCF) versus time after blasts}

Figure 6 shows trends in SCF for each of the blasts. Note the different scales; data are shown for a seven day period following the 1 November 2006 blast, and a 16 day period after the 3 May 2007 blast.

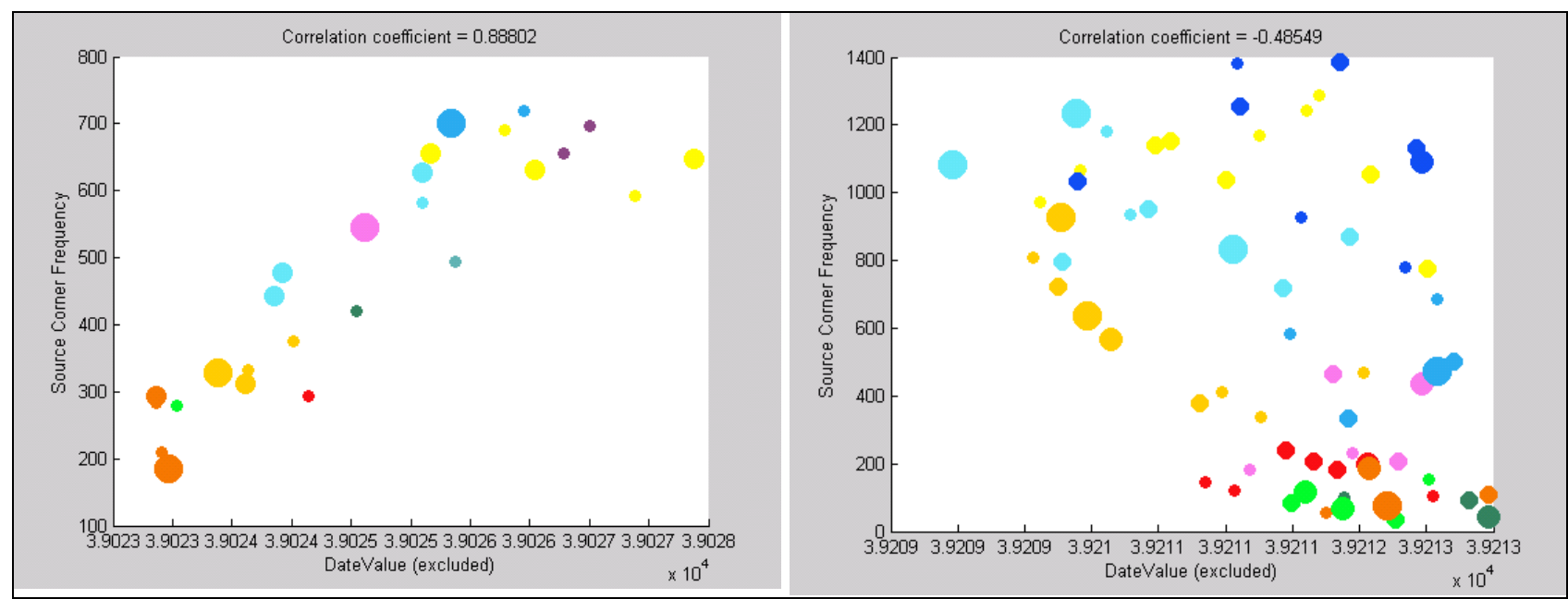

Figure 6 Source corner frequency (SCF) versus time after the blast for (left) the 1 November 2006 blast, and (right) the 3 May 2007 blast. Note: As these results are from different SOM runs, the node colours cannot be directly compared. SCF in $\mathrm{Hz}$

Blast 1 November 2006: The source corner frequency was initially low, and it trended higher over the next seven days. This blast was early in the life of the stoping, and it did not greatly modify the stress field. The increasing SCF is therefore expected.

By reviewing the SOM group colours and the component plots (not shown), additional information is available:

- The initial low SCF events also have a low S:P energy ratio, but high seismic moment. Later in time, the events with higher SCF had high S:P ratios and high stress drops.

- Events with high seismic moment were dominant over those with higher seismic energy, so that overall, moment was the main contributor to magnitude.

- Stress drop correlated well with SCF, S:P energy ratio and seismic moment, but not with energy. 
Blast 3 May 2007: This blast showed an opposite trend. It occurred later in the stoping and caused significant stress changes. A few nodes of high SCF seismic events occurred soon after the blast. They rapidly cascaded into a plethora of events and a variety of nodes.

Additional information obtained from the SOM includes:

- The initial events have high stress drops, moderate/high S:P energy ratio, low moment and some high energies. These initial events continued on for several days (the yellow and dark-blue nodes). The distinction with the 1 November 2006 blast is the higher energies involved.

- Superimposed on the high energy nodes are the dark-yellow nodes; characterised by low S:P energy ratios, low energy and low stress drops.

- After a few days, events appear with low SCF, low S:P energy ratios, but high energy.

Thesis: A tentative thesis could be as follows for initial events following a blast - and clearly this demands further investigation as it bears directly on safety for the underground workforce:

- If seismic moment dominates over energy and the initial S:P energy ratios are lower than usual, the situation is stable and the seismicity will diminish over time (1 November 2006 situation).

- If the initial events have higher than expected energies for their $S C F$, and later events trend to lower S:P ratios, instability is possible and larger seismicity may develop (3 May 2007 situation).

\subsection{Plot of DeltaT vs time after blasts}

Figure 7 shows trends in DeltaT against the time elapsed after the blast. DeltaT is the time difference between an event and its immediate prior event. Again note the different time periods after the blasts (seven days and 16 days respectively).

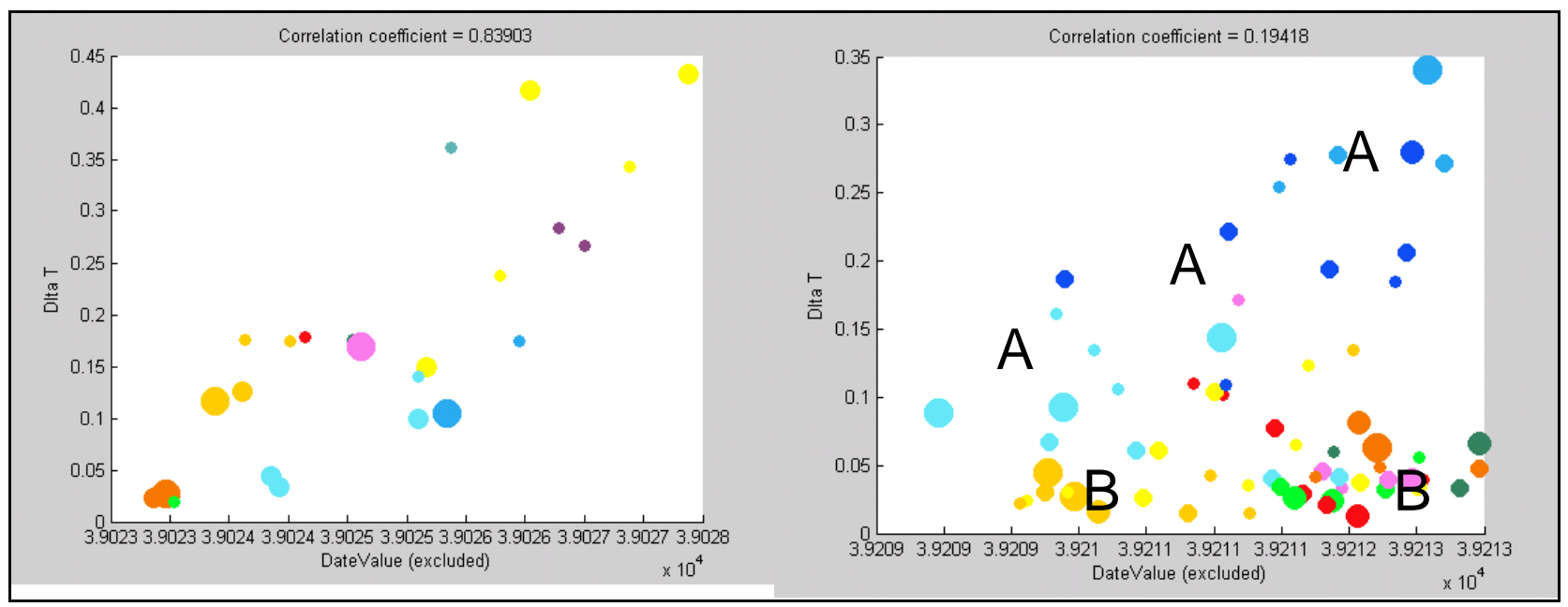

Figure 7 DeltaT (time difference between events) versus time after the blast for (left) the 1 November 2006 blast, and (right) the 3 May 2007 blast. Refer text for explanation of $A$ and B labels. Note: As these results are from different SOM runs, the node colours cannot be directly compared. Time in days

Blast 1 November 2006: DeltaT was initially low, and it trended higher over the seven days. This blast was early in the life of the stoping and it did not greatly modify the stress field. The rapid increase in the time between successive events is therefore, as might be expected. Additional information based on the SOM group colours is:

- Close-timed events have lower S:P energy ratio (this is very characteristic, and seen in other similar blasts besides the one reported here), lower SCF and moderate/high energies.

- Rarer events have high SCF, higher S:P energy ratios, and in this case high stress drops. However, stress drop was variable in other blasts analysed (not shown here). 
Blast 3 May 2007: DeltaT was initially about 0.9 , then spreads out to a wide range of values including very short intervals (i.e. a quiet stope suddenly became active). Additional information, based on the SOM node colours, shows that there are two event classes that perhaps should be called super-groups:

- Super-group A (labelled "A" in Figures 7 and 8): DeltaT for the blue, dark-blue and turquoise nodes steadily increases over time, similar to the 1 November 2006 blast. These are rarer events and are characterised by low P energy, moderate/high S:P energy ratio, high SCF and high stress drops.

- Super-group B (labelled "B" in Figures 7 and 8): DeltaT for the yellow, red and brown nodes, which appear after a day, remains very short. These more common events initially had low $\mathrm{P}$ and $\mathrm{S}$ energies that trended higher, lower S:P energy ratios, high SCF trending rapidly lower, and moderate stress drops becoming lower.

Thesis: A tentative thesis could be as follows:

- In stressed ground, the size and magnitude of seismic events will diminish if events initially have low S:P energy ratios, low SCF and moderate/high energies and if there is a trend over time towards the opposite parameter values.

- The trends in SCF, S:P energy ratio and stress drops, as seen after the 3 May 2007 blast, could be indicators that the ground will remain active for an extended period of time.

A range of additional cross-plots was produced for the 3 May 2007 blast data, of which one is shown in Figure 8. This plot shows a separation of super-groups A and B by magnitude. For a given total energy, the magnitude of the super-group B events is much higher than for the super-group A events. The super-group B events may therefore be the more hazardous. Further study is required to answer questions arising about where these events are in terms of $\mathrm{N}, \mathrm{E}$, Elevation, and whether they might be related to a particular structure.

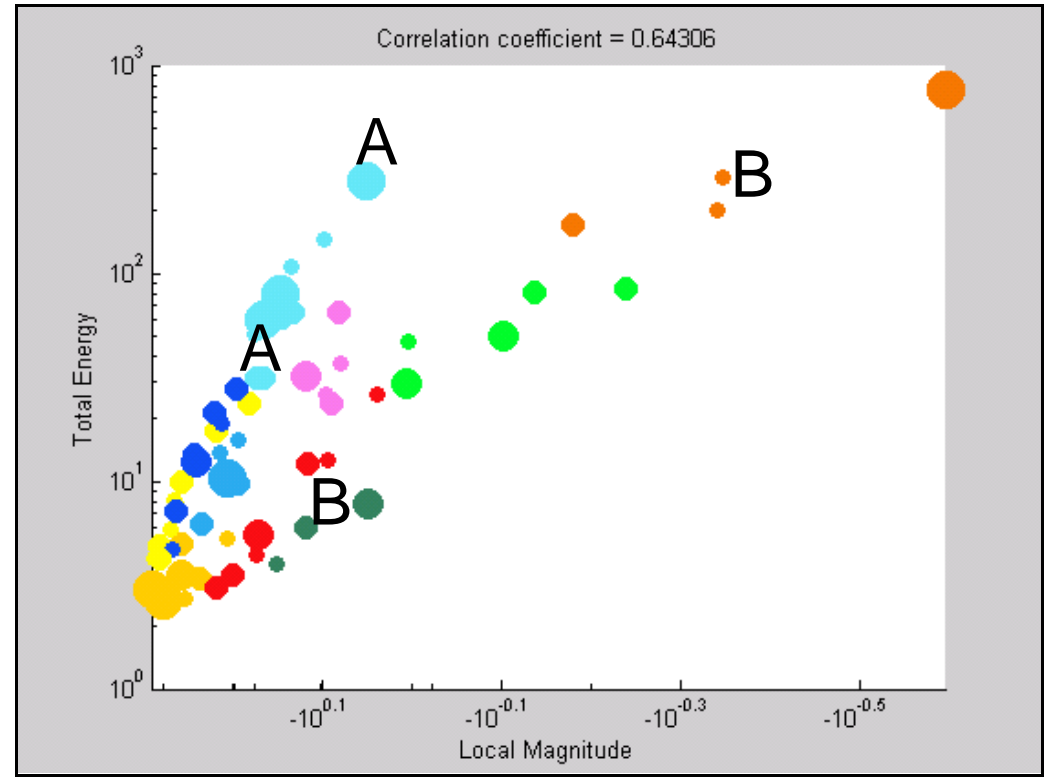

Figure 8 Total energy versus magnitude for the 3 May 2007 blast nodes. Refer text for explanation of $A$ and $B$ labels. Magnitude is approximate Richter. Energy in $\mathbf{J}$

\section{SOM DeltaT analysis of ROB5 seismicity}

Seismic events can occur anywhere, one after another in time, but it is usually assumed that events that occur at about the same time are more related.

This assumption was tested using SOM, by including the DeltaT parameter in the analysis. The full dataset of 6121 events was analysed. DateValue was not included in the SOM analysis (i.e. the dates of events were not analysed in the SOM) but dates were carried through for the purpose of display and further analysis. As 
this was a separate SOM run, the node colours cannot be compared with other plots in this paper. Initial observations are shown by annotation on Figure 9.

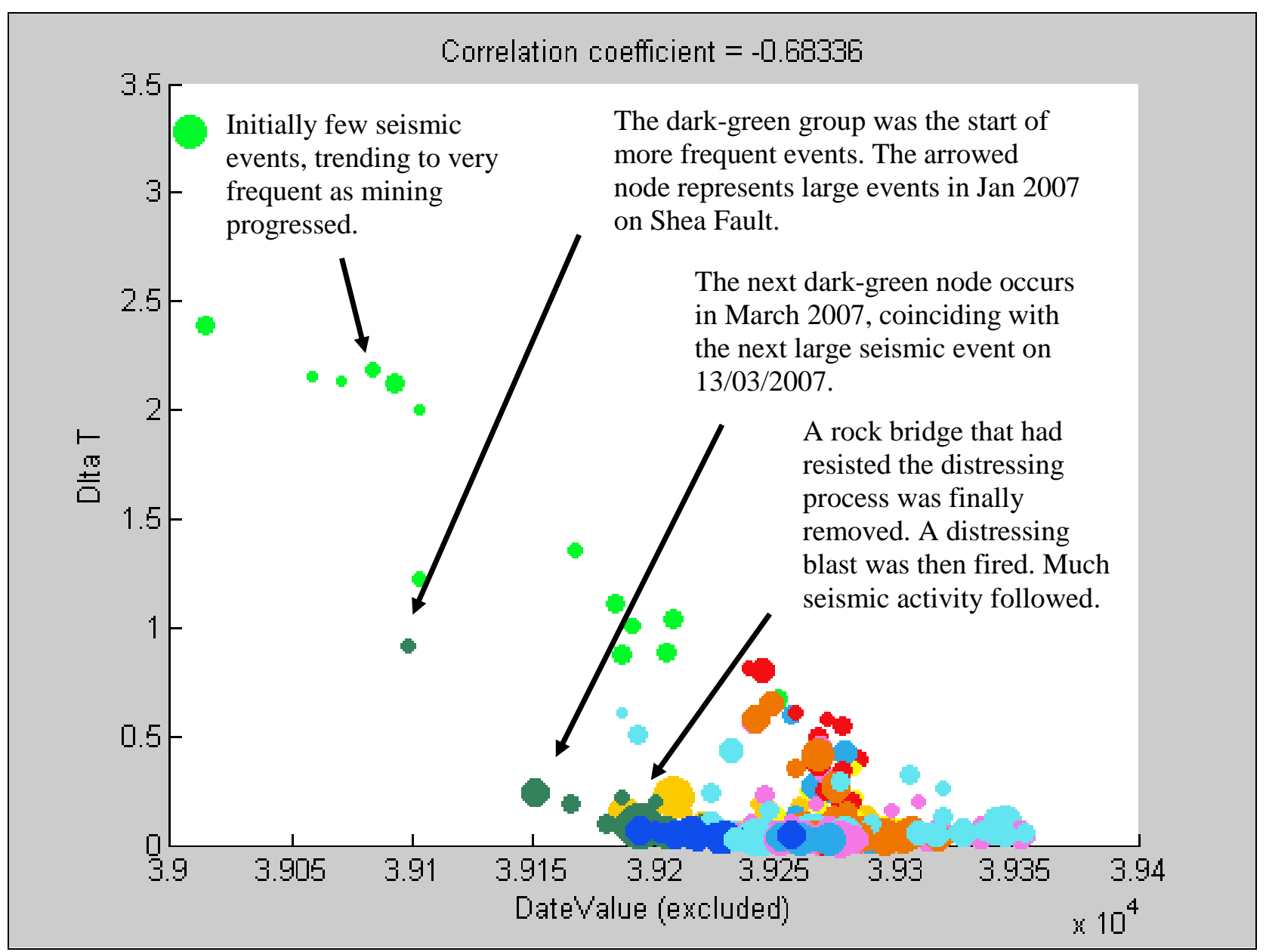

Figure 9 Cross-plot of DeltaT versus DateValue. DeltaT in days. DateValue range covers 400 days from 10 October 2006 to 14 November 2007

DeltaT was further investigated by plotting it against a number of other parameters. The cross-plot for one of these is shown in Figure 10. The nodes and groupings shown in this plot are not pure spatial associations, so they do not locate to a place in the mine. Rather, SOM shows that, wherever they occur, even if hundreds of metres apart, the high-energy events are related in time.

Perhaps in the case of Mount Charlotte, these high-energy events are a function of the time for structure asperities to break, and initiate shearing in response to the associated stress redistributions. Mount Charlotte's structures are typically tight, and are characterised by having offsets at intersections with other structures. This tends to lock structures, preventing movement, until sudden rupture occurs.

The high-energy nodes in Figure 10 occur at characteristic fingerprint times and not others, i.e. this shows that specific lengths of seismic quiescence preceded higher magnitude events during ROB5 mining. This is a very interesting observation. Large unpopulated areas in Figure 10 suggest that DeltaT can be a guide to possible event energies. For ROB5 mining:

- There was a favourite average DeltaT for the highest energy events of 0.25 days (six hours).

- There was an increasing DeltaT for larger event energies.

- All of the high-energy events had the characteristics of the dark-yellow group. 
Other cross-plots (not shown here) reveal a similar seismic quiescence period in relation to average seismic moment. There is clearly more work to do to investigate this dark-yellow group; to establish where these events are spatially, whether they are related to a specific structure orientation, and why.

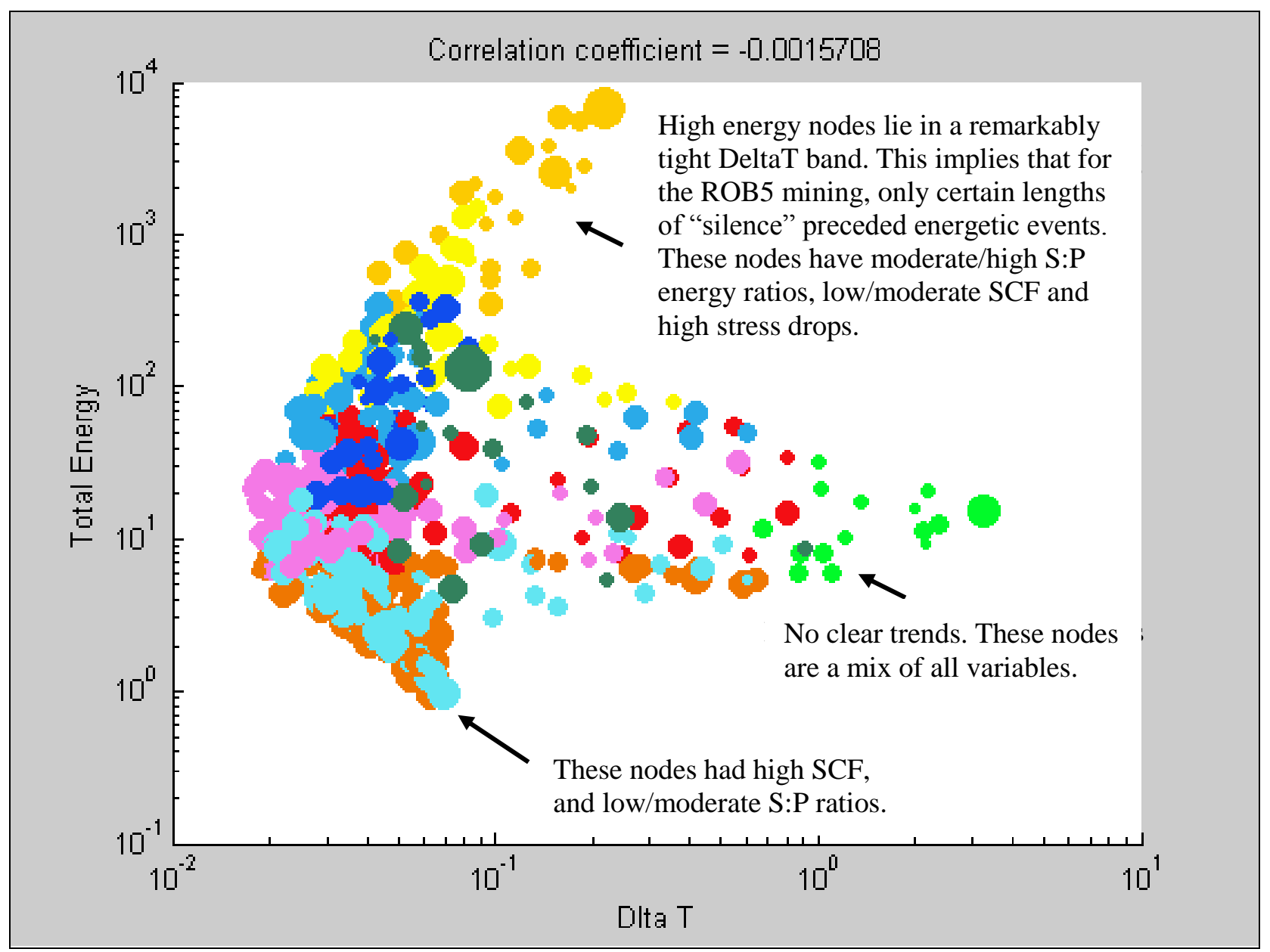

Figure 10 Cross-plot of DeltaT versus total energy DeltaT in days; energy in J

\section{Conclusions}

Programs such as MS-RAP have come a long way in the interpretation of spatial event groups. From that starting point it is difficult, conceptually, not to think of SOM groups as spatial, as being near a particular structure or void. Yet this difficulty is SOM's strength, as it opens a door to the study of multi-parameter groups. Instead of one clustering basis, SOM can allow dozens of ways of analysing the same dataset.

Many more observations and questions arose from querying the SOM data than could be discussed in this paper. These preliminary results are sufficiently encouraging to pursue this technique in greater depth for the analysis of the ROB5 seismic dataset.

Of particular interest to Mount Charlotte is the use of SOM to investigate relationships between seismic events and geology; particularly relationships with faults and structures of particular orientations. Relationships to the tonnage broken per blast (as an estimate of the significance of the blast) will also be of interest.

As always, however, all seismic analyses require good datasets, to ensure that the parameters being analysed are potentially meaningful. The ROB5 dataset proved to be a good choice for analysis in this regard. Practitioners are therefore encouraged to be rigorous about data collection and protection.

SOM adds more dimensions to the analysis of seismic data. In some aspects it is complementary to existing methods such as MS-RAP, but in others it is quite novel. SOM would also provide greater insight into other 
complex Earth Science data sets. It will no doubt challenge our current knowledge, but it may also improve our understanding of complex multi-parameter situations.

\section{Acknowledgements}

The authors gratefully acknowledge KCGM and CSIRO for permission to publish this paper, and the support and probing questions, over many years, of Mount Charlotte's mine managers, engineers and operators.

\section{References}

Fraser, S.J. (2004) Proposal for Pilot Study into the Effectiveness of "CSOM" as a Predictive Tool for Forecasting Seismicity at the Mine Design Stage at Mount Charlotte, Kalgoorlie, Western Australia, CSIRO Exploration and Mining, Brisbane, November.

Fraser, S.J., Mikula, P.A., Lee, M.F., Dickson, B.L. and Kinnersly, E. (2006) Data Mining Mining Data: Ordered Vector Quantization and its Application to Mine Geotechnical Data Sets, 6th International Mining Geologists Conference, Darwin, August.

Hudyma, M., Potvin, Y. and Heal, D. (2007) The Mine Seismicity Risk Analysis Program (MS-RAP) - Transforming Microseismic Data into Rock Engineering Knowledge. Y. Potvin, J. Hadjigeorgiou, T.R. Stacey (editors), Challenges in Deep and High Stress Mining, Australian Centre for Geomechanics, Perth, pp. 427-434.

Kempin, M., Sprague, A., Narendranathan, S., Mikula, P.A. and Lee, M.F. (2007) Destressing the ROB5 Remnant using Tight Slot Blasting at Mount Charlotte Mine, 4th International Seminar on Deep and High Stress Mining, Australian Centre for Geomechanics, Perth, November.

Kohonen, T. (2001) Self-Organising Maps: Third Extended Edition, Springer Series in Information Sciences, Vol. 30, Springer, Berlin, Heidelberg, New York.

Mikula, P.A. (2006) ROB5 Geotechnical Mining Strategy, Mikula Geotechnics Report to Kalgoorlie Consolidated Gold Mines, Mount Charlotte Operations, May.

Mikula, P.A. and Lee, M.F. (2002) Forecasting and Controlling Pillar Instability at Mount Charlotte Mine, Deep and High Stress Mining - First International Seminar, Australian Centre for Geomechanics, November.

Mikula, P.A., Sharrock, G., Lee, M.F. and Kinnersly, E. (2005) Seismicity Management Using Tight Slot Blasting for Stress Control at Mount Charlotte Mine, 6th International Symposium on Rockburst and Seismicity in Mines, Australian Centre for Geomechanics, Perth.

Sharrock, G. (2004) Seismic Analysis of ROB5 Destressing, AMC Consultants Report No 204048 for Kalgoorlie Consolidated Gold Mines, Mount Charlotte Operations, June. 